\title{
Probing Red Giant Atmospheres with Gravitational Microlensing
}

\section{Citation}

Heyrovsky, David, Dimitar Sasselov, and Abraham Loeb. 2000. "Probing Red Giant Atmospheres with Gravitational Microlensing." The Astrophysical Journal 543 (1): 406-16. https:// doi.org/10.1086/317067.

\section{Permanent link}

http://nrs.harvard.edu/urn-3:HUL.InstRepos:41393268

\section{Terms of Use}

This article was downloaded from Harvard University's DASH repository, and is made available under the terms and conditions applicable to Other Posted Material, as set forth at http:// nrs.harvard.edu/urn-3:HUL.InstRepos:dash.current.terms-of-use\#LAA

\section{Share Your Story}

The Harvard community has made this article openly available.

Please share how this access benefits you. Submit a story.

Accessibility 


\title{
Probing Red Giant Atmospheres with Gravitational Microlensing
}

\author{
David Heyrovský, Dimitar Sasselov and Abraham Loeb \\ Department of Astronomy, Harvard University \\ 60 Garden St., Cambridge, MA 02138, USA
}

\begin{abstract}
Gravitational microlensing provides a new technique for studying the surfaces of distant stars. Microlensing events are detected in real time and can be followed up with precision photometry and spectroscopy. This method is particularly adequate for studying red giants in the Galactic bulge. Recently we developed an efficient method capable of computing the lensing effect for thousands of frequencies in a high-resolution stellar spectrum. Here we demonstrate the effects of microlensing on synthesized optical spectra of red giant model atmospheres. We show that different properties of the stellar surface can be recovered from time-dependent photometry and spectroscopy of a point-mass microlensing event with a small impact parameter. In this study we concentrate on center-to-limb variation of spectral features. Measuring such variations can reveal the depth structure of the atmosphere of the source star.
\end{abstract}

Subject headings: gravitational lensing — red giants — stars: atmospheres

Submitted to The Astrophysical Journal, February 1999 


\section{Introduction}

Stars populating the red giant branch spend a substantial fraction $(\geq 5 \%)$ of their entire lifetime there. Red giants are thus a major component of any galactic stellar population. In old systems, like Galactic globular clusters, their contribution is even larger. The parameters of the red giant branch have been traditionally derived from multi-color photometry and interpreted using stellar atmosphere models (e.g. Vandenberg \& Bell 1985; Kurucz 1992). These model atmospheres involve one-dimensional integrations of semi-infinite slabs in hydrostatic and radiative equilibrium. However, real atmospheres of red giants are usually extended and the slab approximation is not adequate. Despite significant progress in computing extended spherically-symmetric models (e.g., Scholz \& Tsuji 1984; Plez, Brett, \& Nordlund 1992; to name a few), it is still difficult to use these improvements in massive grids of models, which therefore continue to be computed as plane-parallel (Houdashelt et al. 2000). A number of other assumptions and simplifications had to be usually made, some of which have not been verified yet by direct observations. Examples include the treatment of convective transport and small-scale velocity fields (e.g. micro- and macro-turbulence). Similarly, stellar evolution models also depend on a number of parameters which describe poorly understood physics, such as convection near the surface of the star.

These problems have hindered significant improvement in the determination of fundamental stellar properties over the past two decades. The angular diameters of only a small number of stars have been measured up to now, with an accuracy of $\sim 10 \%$ at best (Armstrong et al. 1995). Stellar disk brightness distributions (limb darkening) have been inferred only for a handful of stars at the level of a proof-of-concept (directly Mozurkewich et al. 1991; or in binaries - Andersen 1991). An exception is provided by the direct $H S T$ observation of the red supergiant $\alpha$ Ori, which revealed limb darkening as well as a bright region on the surface (Uitenbroek, Dupree, \& Gilliland 1998). In general, the available inferred results cannot be used to build a model atmosphere, as in the case of the Sun. Due to this lack of an adequate observational basis, our understanding of stellar light is implicitly tied to the solar atmosphere model.

This situation is particularly unfortunate, since recently there has been an increased demand for accurate stellar models in a number of applications. For example, much improved color-temperature conversions are needed for calibrating distance indicators and determining stellar ages. Stellar population syntheses (e.g. for high-redshift galaxies) also rely critically on the accuracy of current stellar models. Our Sun is not a good standard for most of these applications. 
The advent of large interferometric arrays (Keck interferometer, CHARA, SIM, VLTI) will go a long way towards solving these problems, but these complex and expensive facilities are still mostly in a preliminary development phase. In the meantime, gravitational microlensing offers an easily accessible, immediate, and inexpensive means for imaging at least large stars, such as red giants. It also offers, by its nature, access to stellar populations in the Galactic bulge and the Magellanic Clouds, which are well beyond the reach of any interferometer.

By now there are more than 350 microlensing events detected towards the Galactic bulge and the Large and Small Magellanic Clouds (see e.g. Alcock et al. 1997a, 1997b, 1997c; Becker et al. 1998; Renault et al. 1997; Afonso et al. 1999; Udalski et al. 1997). During a Galactic gravitational microlensing event the flux from a distant star is temporarily amplified by the gravitational field of a massive dim object passing in the foreground. The standard light curve in the point-source limit is characterized by two observables: its peak amplitude and duration (Paczyński 1996). These observables physically depend only on the lensing impact parameter and the Einstein radius crossing time of the source. However, in events with a small impact parameter the light curve is also affected by the resolved surface of the lensed star, thus providing an excellent opportunity for stellar surface imaging (Valls-Gabaud 1995, Sasselov 1996).

The first well documented case of resolved finite-size microlensing effects is MACHO Alert 95-30 (hereafter M95-30), with the impressive follow-up campaign by the GMAN and MACHO collaborations (Alcock et al. 1997d). The first spectroscopic observations of a binary lens event in which the caustic crossed the face of the source star were reported by Lennon et al. (1996). In a similar caustic-crossing binary event, Albrow et al. (1999) recently determined limb darkening profiles of the lensed $\mathrm{K}$ giant star for both observed spectral bands $(I$ and $V)$.

Finite-size effects were first theoretically studied as methods to partially remove the degeneracy of microlensing light curves. These methods are based on alterations of the standard light curve (e.g. Gould 1994, 1995; Nemiroff \& Wickramasinghe 1994; Witt \& Mao 1994; Gould \& Welch 1996), resolved polarization (Simmons, Willis, \& Newsam 1995; Simmons, Newsam, \& Willis 1995; Bogdanov, Cherepashchuk, \& Sazhin 1996), spectral shifts due to stellar rotation (Maoz \& Gould 1994), and narrow-band photometry of resonance lines (Loeb \& Sasselov 1995). Here we want to put emphasis on the inverse problem - using microlensing for studying stellar surface features and probing the atmosphere of the source star.

The resolved stellar surface brightness distribution, $B(\lambda, \vec{r})$, can vary strongly with wavelength in selected spectral regions - in the continuum as well as within spectral 
lines. The time-dependent microlensing amplification then becomes wavelength-dependent through $B(\lambda, \vec{r})$. Photometry in different bands and sets of spectra taken in the course of a microlensing event can therefore be used to study the brightness distribution of the source. Microlensing light curve chromaticity was first evaluated by Witt (1995), Valls-Gabaud (1995) and Bogdanov \& Cherepashchuk (1995). Methods for obtaining the brightness distribution by light curve inversion were studied by Bogdanov \& Cherepashchuk (1996) and Hendry et al. (1998), inversion error analysis was presented by Gaudi \& Gould (1999). Few works have been published so far on spectral effects in stellar microlensing. These include studies of line profile changes in rotating giants (Maoz \& Gould 1994; Gould 1997) and in circumstellar envelopes in bulk motion (Ignace \& Hendry 1999). First results for selected spectral lines in a simple model stellar atmosphere were presented by Valls-Gabaud (1996, 1998).

In order to optimize observations of spectral effects in actual events, the most practical approach is to theoretically predict the lensing effect on the entire synthesized optical spectrum of the source in microlensing alerts similar to M95-30. This way one can determine the most sensitive spectral features before the event peaks. An efficient method and computer code capable of dealing with the tens of thousands of frequencies involved was developed by Heyrovský \& Loeb (1997; hereafter Paper I). In this work we use the method to explore point-mass microlensing effects on both low and high resolution optical spectra, synthesized directly from up-to-date red giant model atmosphere calculations.

The outline of this paper is as follows. In $\S 2$ we describe the model atmospheres used in this paper. The effect of microlensing on the overall spectrum of the source star as well as on individual spectral features is presented in $\S 3$. Finally $\S 4$ summarizes the main conclusions and future prospects of this work.

\section{Model Atmospheres of Red Giants}

In a stellar population with some spread of metallicities red giants exhibit a range of temperatures. The cooler giants have atmospheres which, unlike in the case of the Sun, are dominated by molecular compounds. Most of the hydrogen gets locked into $\mathrm{H}_{2}$ and most of the carbon into CO. As the temperature drops, TiO, VO, and eventually $\mathrm{H}_{2} \mathrm{O}$ become

prominent. This requires paying special attention to the equation of state and background opacities (Hauschildt et al. 1997; Kurucz 1992). Of great importance is the treatment of convection, as transport by convection affects a large part of the photosphere. Finally, for 

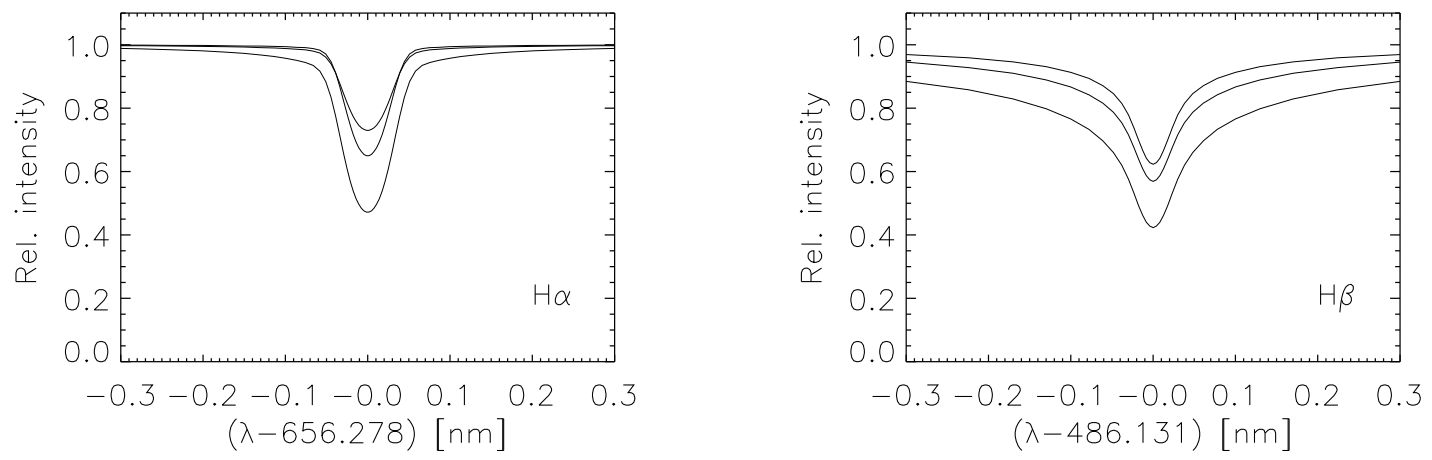

Fig. 1.- NLTE line profiles from different positions on the stellar disk in a red giant model with $\mathrm{T}=3750 \mathrm{~K}, \log g=0.5$. Left panel: profiles of the hydrogen $\mathrm{H} \alpha$ line from disk center at $\mu=0.996$ (lowest curve), through $\mu=0.5$ (central curve at line core), to very near the limb at $\mu=0.004$ (upper curve at line core). Here $\mu$ is the cosine of the angle between the normal to the stellar surface and the line of sight (referred to as "the incidence angle" hereafter). Right panel: same for the hydrogen $\mathrm{H} \beta$ line; the profile sequence is $\mu=0.996$ (lowest curve), $\mu=0.004$ (central curve) and $\mu=0.5$ (upper curve). Note the difference in profile shape, as well as in its change with $\mu$.

models with high luminosity (and low surface gravity, $\log g \leq 1$ ), spherical geometry must be adopted in the treatment of radiative transfer (Hauschildt et al. 1999).

Here we have limited our study to static (i.e., non-Mira) red giant models. Our main interest is to compute realistic models of the center-to-limb variation of the intensity on the stellar disk - both for individual spectral features in high resolution, as well as for the optical pseudo-continuum. The state-of-the-art work for such models has been done recently by Hofmann \& Scholz (1998) and Jacob et al. (1999). They employ model atmospheres from the grid of Bessell et al. $(1989,1991)$. Here we use similar models which reproduce their results. Striving for this level of sophistication is crucial in our study of center-to-limb and depth dependence of spectral features in red giants. For example, Valls-Gabaud (1998) used the linear limb darkening law and fitted simple analytical expressions for spectral lines from Kurucz (1992) model grids to predict chromatic and spectroscopic signatures of microlensing events of stars in general. We could not use the same simple approach, because we find that for red giants these linear approximations fail by a factor which is much larger than the uncertainties of the models, in line with similar findings by Jacob et al. (1999).

The structure of the models in this paper is derived by assuming local thermodynamic equilibrium (LTE) for the background opacities in flux-constant plane-parallel atmospheres. 

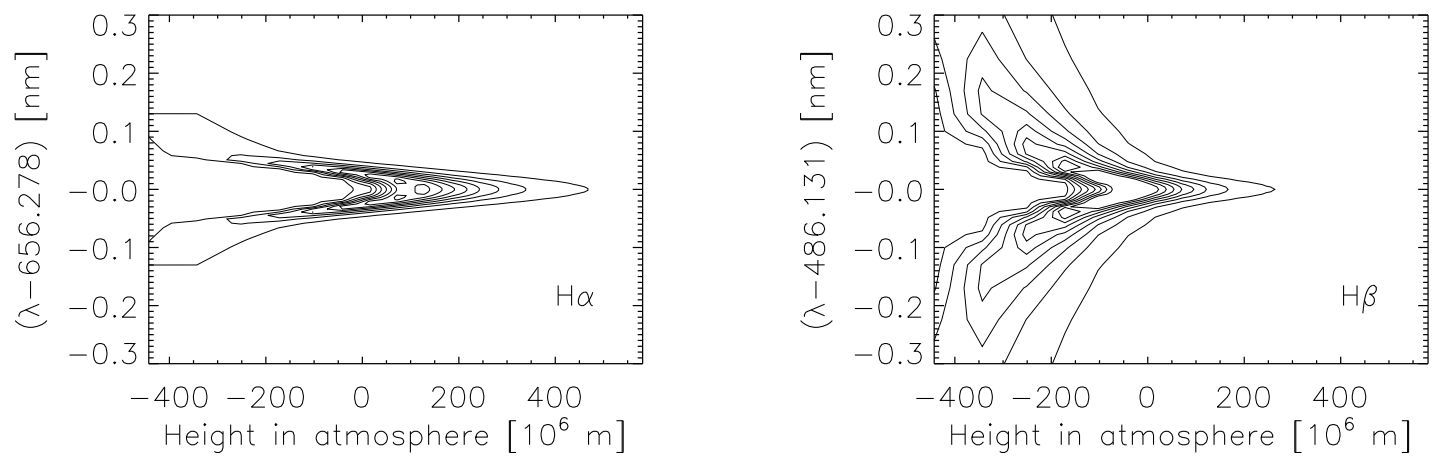

Fig. 2.- Line-forming regions of the hydrogen $\mathrm{H} \alpha$ and $\mathrm{H} \beta$ lines shown in Figure 1, represented by their contribution functions (CFs). The CF contours range from 0.1 (outermost) to 1.5 (innermost) in units of normalized intensity. Left panel: the hydrogen $\mathrm{H} \alpha$ line; right panel: the hydrogen $\mathrm{H} \beta$ line. Zero height is defined at continuum optical depth of unity for the rest wavelength of each line respectively.

We use the most recent opacity sampling routines of Kurucz (1999) and TiO line data of Schwenke (1998). These models serve as input for computations of hydrogen and calcium, which are treated out of LTE (NLTE) with multi-level model atoms and in spherical geometry (for more details see Loeb \& Sasselov 1995). The hydrogen atom model has energy levels $n=1-5$, plus continuum; all bound-bound and bound-free transitions are calculated in detail. The calcium atom model contains 8 energy levels for the neutral species (CaI) and 5 energy levels for the first ionization state (CaII), plus continuum (CaIII); all transitions are calculated in detail. In the case of red giants, atomic hydrogen and calcium are not important for the overall atmospheric structure. However, they still provide strong spectral features with significant center-to-limb variation (e.g. in the hydrogen Balmer lines, CaII H\&K, etc.). Most moderately strong and weak lines diminish towards the limb (see Figure 1). As discussed further below, this variation could be detected in a microlensing event by measuring the total equivalent widths of spectral lines on medium-resolution spectra with a high signal-to-noise ratio, or it could be seen directly on high-resolution spectra (see $\S 3)$.

The variation of the intensity from the center of the stellar disk to its limb is studied theoretically by computing the contribution function of each transition. This function defines the line-forming region in both space (depth in the atmosphere) and frequency. The line-forming region is shaped by local as well as non-local influences. Following Magain (1986), we define the NLTE contribution function (CF) for the relative line 
depression as

$$
C F\left(\log \tau_{\lambda_{0}}\right)=\frac{\ln 10}{\mu} \tau_{\lambda_{0}} \frac{\kappa_{l}}{\kappa_{0}}\left(1-\frac{S_{l}}{I_{c}}\right) e^{-\tau_{R} / \mu}
$$

where $\mu$ is the cosine of the incidence angle; $\tau_{\lambda_{0}}$ is the optical depth at a reference wavelength $\lambda_{0} ; \tau_{R}$ is the optical depth corresponding to the opacity $\kappa_{R}=\kappa_{l}+\kappa_{c} S_{c} / I_{c} ; \kappa_{0}$ is the absorption coefficient at $\lambda_{0} ; \kappa_{l}$ and $\kappa_{c}$ are the line and continuum absorption coefficients, respectively; $S_{l}$ and $S_{c}$ are the line and continuum source functions, respectively; and $I_{c}$ is the emergent continuum intensity (if the line were absent). The traditional dependence on frequency has been omitted from our notation for clarity. The CF for a given synthesized spectral line indicates where the line is formed, and thus it provides a theoretical tool for recovering the depth dependence of thermodynamic variables in stellar atmospheres.

The CFs of the two lines presented in Figure 1 are shown in Figure 2. Note how two spectral lines from the same element and spectral series can exhibit differences in their CFs, in the CF's location against the local continuum, and hence in their center-to-limb variation. For example, the change in the strength of the $\mathrm{H} \beta$ line undergoes a reversal close to the limb of the star. The line gradually weakens away from the disk center until it reaches a minimum, then it becomes slightly more prominent again near the limb. In the case of the $\mathrm{H} \alpha$ line this effect is much weaker and occurs closer to the limb. This difference between the two lines is due to the stronger dependence of $\mathrm{H} \beta$ on the $\mathrm{TiO}$ opacity, which is dominant at its wavelength.

\section{Effects of Microlensing on the Source Spectrum}

\subsection{Microlensing of an Extended Source}

Next we study the effect of microlensing by a point-mass on the spectrum of the source star. For simplicity we describe the lensing configuration in terms of angular distances in the plane of the sky, using the angular source radius as a length unit.

In the absence of a lens, the observed flux from an unresolved source star at wavelength $\lambda$ is obtained by integrating the surface brightness distribution $B(\lambda, \vec{r})$ over the projected surface of the star $\Sigma$,

$$
F_{0}(\lambda)=\int_{\Sigma} B(\lambda, \vec{r}) d \Sigma
$$


Any information about the surface structure is therefore concealed by the integral. This information can be uncovered by microlensing, as its signature contains differential information on the brightness distribution. The flux from a simple point-source separated from a point-mass lens by a projected distance $\sigma$ in the plane of the sky gets amplified by a factor

$$
A_{0}(\sigma)=\frac{\sigma^{2}+2 \epsilon^{2}}{\sigma \sqrt{\sigma^{2}+4 \epsilon^{2}}},
$$

where $\epsilon$ is the angular Einstein radius of the lens (Paczyński 1996). The lensed flux from an extended source can then be computed as

$$
F\left(\lambda, \sigma_{0}\right)=\int_{\Sigma} B(\lambda, \vec{r}) A_{0}(\sigma) d \Sigma,
$$

here $\sigma$ is the distance from the lens to point $\vec{r}$ on the source, $\sigma_{0}$ is the distance to the source center. In our units, $\sigma_{0}=1$ corresponds to having the lens projected at the source limb. The conversion between the $\sigma$ distances and the source-centered position vector $\vec{r}=(\rho \cos \psi, \rho \sin \psi)$ is

$$
\sigma=\sqrt{\sigma_{0}^{2}-2 \sigma_{0} \rho \cos \psi+\rho^{2}}
$$

The coordinates are oriented so that the lens lies in the direction $\psi=0$. In this notation $\rho=\sqrt{1-\mu^{2}}$, where $\mu$ is the cosine of the incidence angle used in $\S 2$. Generally the flux in equation (3) depends also on the angle between the lens and a fixed direction on the source (see Paper I). However, in this work we consider for simplicity a circularly symmetric source with $B(\lambda, \vec{r})=B(\lambda, \rho)$. Angular surface irregularities such as spots are discussed in detail in a separate paper (Heyrovský \& Sasselov 2000a). Equation (3) indicates that as the lens moves, it gradually scans the source, giving highest weight to the region of the star closest to the projected lens position. The total resulting amplification of the source star is

$$
A\left(\lambda, \sigma_{0}\right)=\frac{F\left(\lambda, \sigma_{0}\right)}{F_{0}(\lambda)} .
$$

The center-to-limb surface brightness profile ( $B$ as a function of $\rho$ ) at different wavelengths has not only a different amplitude, but also a different shape. As a consequence, the wavelength- and position-dependence of the brightness distribution cannot be separated $B(\lambda, \rho) \neq f_{1}(\lambda) f_{2}(\rho)$ and equation (5) truly contains a chromatic dependence of the amplification. This fact does not contradict the achromaticity of light deflection predicted by the general theory of relativity, it merely reflects the different appearance of the source star at different wavelengths.

For most microlensing events the point-source limit in equation (2) gives a satisfactory description of the light curve. Extended source effects become important only in events with 
a sufficiently low impact parameter (defined as the projected distance of closest approach of the lens and source on the sky). Expanding the amplification (5) in powers of the inverse of the lens-source separation $\sigma_{0}^{-1}$ irrespective of the Einstein radius of the lens (keeping the ratio $\epsilon / \sigma_{0}$ fixed) we obtain

$$
A\left(\lambda, \sigma_{0}\right)=\frac{\sigma_{0}^{2}+2 \epsilon^{2}}{\sigma_{0} \sqrt{\sigma_{0}^{2}+4 \epsilon^{2}}}\left[1+\frac{8 \epsilon^{4}\left(\sigma_{0}^{2}+\epsilon^{2}\right)}{\sigma_{0}^{2}\left(\sigma_{0}^{2}+2 \epsilon^{2}\right)\left(\sigma_{0}^{2}+4 \epsilon^{2}\right)^{2}} \frac{\int_{0}^{1} B(\lambda, \rho) \rho^{3} d \rho}{\int_{0}^{1} B(\lambda, \rho) \rho d \rho}+o\left(\sigma_{0}^{-2}\right)\right] .
$$

Strictly speaking, this $\sigma_{0} \gg 1$ expansion is convergent for $\sigma_{0}>1+\sqrt{2}$. The first extended source correction term in the brackets is a product of two factors. The first one depends purely on the lens parameters $\left(\epsilon, \sigma_{0}\right)$, while the second one depends only on the source properties $(B)$. The lens factor is a monotonously decreasing function of the lens distance, dropping from a value of $\sigma_{0}^{-2} / 4$ when the source lies well within the Einstein $\operatorname{ring}\left(\sigma_{0} \ll \epsilon\right)$ to a value of $8 \epsilon^{4} \sigma_{0}^{-6}$ when the source is well beyond the Einstein ring $\left(\sigma_{0} \gg \epsilon\right)$. The magnitude of the chromatic source factor is less than one, typically on the order of 0.4 . Using this value we find that any lens with $\epsilon \gtrsim 4$ will produce a $1 \%$ effect at $\sigma_{0}=3$. Therefore, in order to achieve a larger extended source effect, even a lens with high $\epsilon$ has to approach the source closer than 3 source radii, beyond the region where the above expansion is valid . An observed example of such an event is M95-30 (Alcock et al. 1997d), with a fitted value of the impact parameter $p=0.715 \pm 0.003$ and $\epsilon=13.23 \pm 0.02$.

Note that we estimate the extended source effect in comparison to a point-source light curve with the same event parameters. Studying detectability requires comparison with a best-fit point-source light curve, which further lowers the upper limit for the closest approach. Gould \& Welch (1996) estimate that using color observations the finite-source effect is detectable out to 2 source radii.

We study the case of close approaches or source transits directly from equations (3) and (5). For evaluation of the involved surface integral we follow the method described in Paper I. The $\sigma^{-1}$ divergence of the amplification factor $A_{0}(\sigma)$ at the point directly behind the lens can be avoided by integrating in lens-centered polar coordinates. The product of $A_{0}(\sigma)$ with the area element $d \Sigma=\sigma d \sigma d \phi$ then leads to a finite integrand. For $B(\lambda, \rho)$ we use brightness profile data generated for each wavelength directly from model atmosphere calculations (see $\S 2$ ). We interpolate the computed center-to-limb data points by simple quadratic segments $a+b \rho^{2}$. The computational advantage of this approach is

1 Lenses with $\epsilon \lesssim 0.4$ are too weak to trigger a microlensing alert in the current surveys. Lenses with $0.4<\epsilon<4$ require an approach closer than 3 source radii for a $1 \%$ effect. 

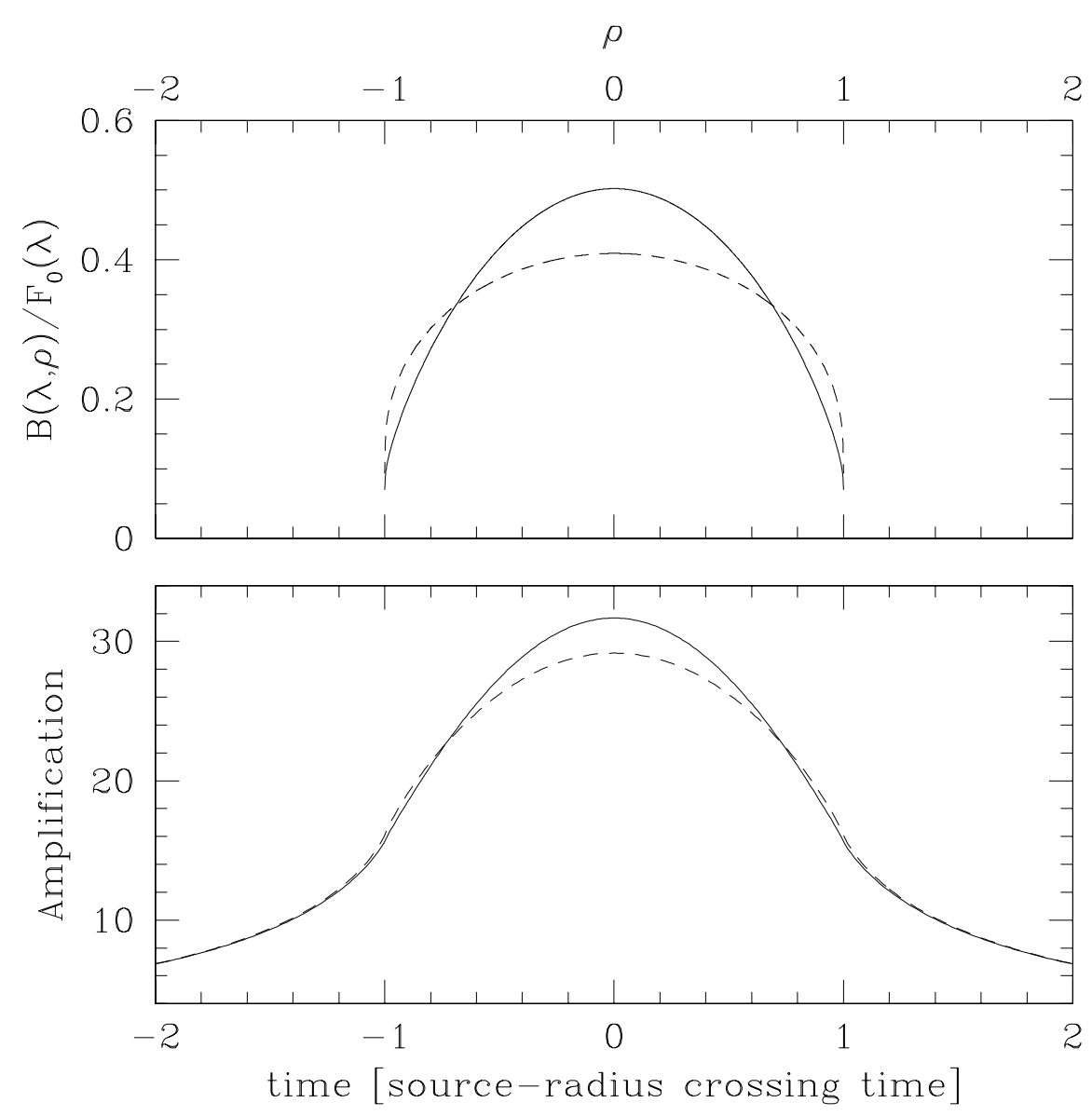

Fig. 3.- Lower panel: light curves for two selected wavelengths (451 nm - solid line, $759 \mathrm{~nm}$ dashed line) of a $\mathrm{T}=3750 \mathrm{~K}, \log g=0.5$ model atmosphere (spectral resolution as in Figure 4 ), lensed by an $\epsilon=13.23$ lens with zero impact parameter. Upper panel: corresponding normalized surface brightness profiles. For the purpose of alignment with the lower panel the profiles are reflected to negative values of $\rho$.

that the integral can then be analytically reduced to one dimension (for details see Paper I). Additionally, as the lensed flux is linear in the interpolation coefficients, it is sufficient to compute the light curve for a single wavelength and then by substituting coefficients obtain the other light curves for the same event. With this technique it is feasible to perform the computation for a large number of wavelengths and thus study spectral changes associated with microlensing.

\subsection{Light Curve Shapes and Low Resolution Spectra}




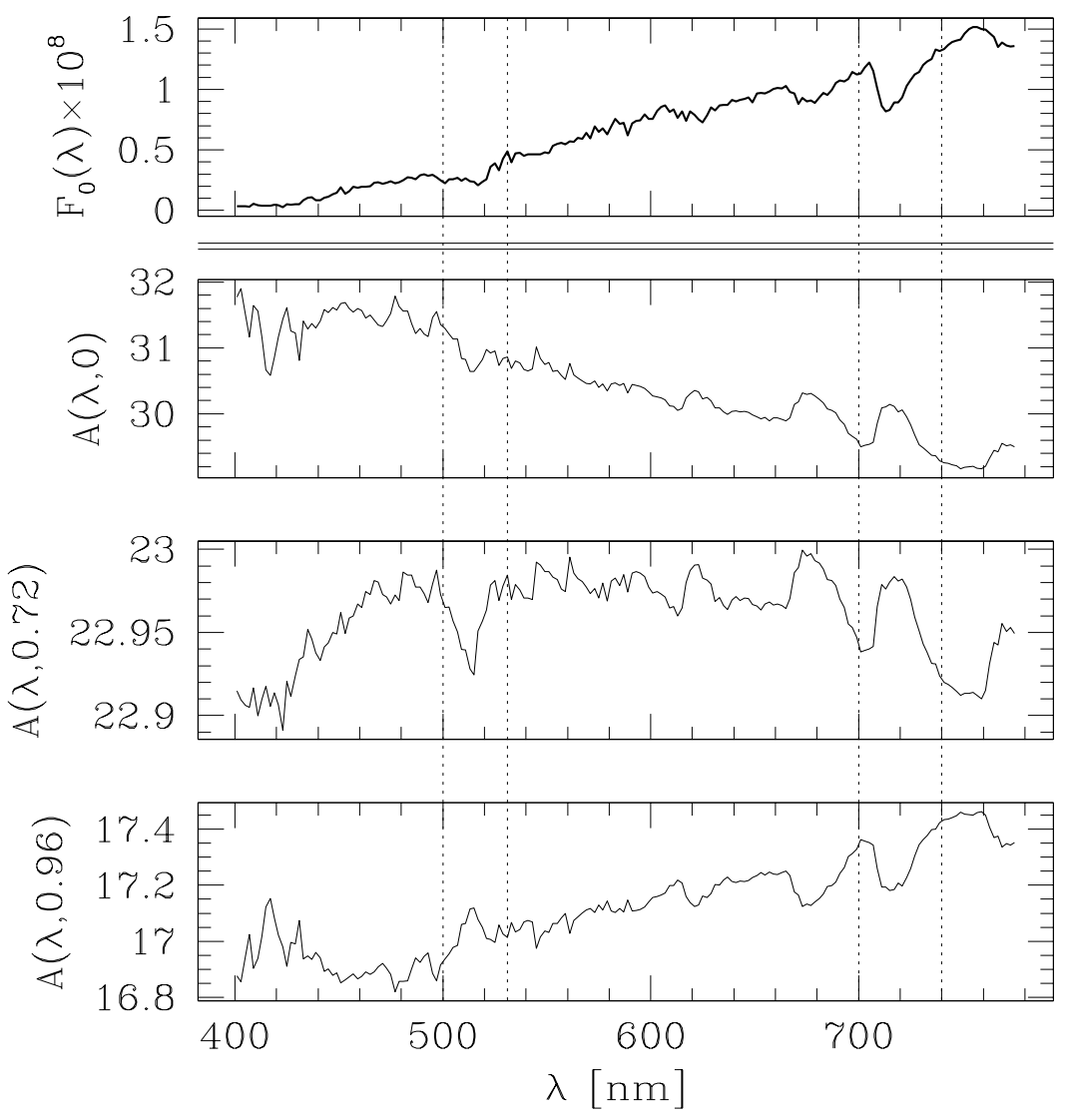

Fig. 4. - Upper panel: low resolution unlensed spectrum of the $\mathrm{T}=3750 \mathrm{~K}, \log g=0.5$ model atmosphere (flux in units of $W \mathrm{~m}^{-2} \mathrm{~Hz}^{-1}$ ). Lower three panels: amplification by an $\epsilon=$ 13.23 lens as a function of wavelength for lens positions $\sigma_{0}=0,0.72$ and 0.96 . Vertical dotted lines mark two regions with $\mathrm{TiO}$ bands affected by microlensing in opposite ways. These regions are enlarged with high spectral resolution in Figure 8 .

The effect of the brightness profile shape on the light curve is demonstrated in Figure 3 . For both selected wavelengths the profile is normalized to unit total flux, in order to match the form in which it appears in the amplification formula (5). The light curves plotted in the lower panel have a zero impact parameter, thus the time $t$ measured from closest approach in units of source radius crossing time is equal to the lens distance $\left(|t|=\sigma_{0}\right)$. For illustration we use the M95-30 lens with $\epsilon=13.23$. From the figure it can be seen that the light curve traces the brightness profile shape - the flatter profile produces a flatter light curve. In addition, the light curve with lower amplification at the source center typically crosses the other at $\sigma_{0} \sim 0.7$ and has a higher amplification at the limb of the source. We note that flatter profiles usually indicate formation higher in the stellar atmosphere. 
Figure 1 illustrates the wavelength dependence of the amplification on a low resolution $(\mathrm{R}=500)$ spectrum from 401 to $775 \mathrm{~nm}$. These results are obtained using the same model atmosphere as in Figure 3, with $\mathrm{T}=3750 \mathrm{~K}$ and $\log g=0.5$, lensed by an $\epsilon=13.23$ lens. The model spectrum in the absence of the lens is shown in the upper panel. The lower three amplification plots correspond to lens positions $\sigma_{0}=0$ (source center), 0.72 (closest approach of M95-30) and 0.96 (close to the limb). Comparison of the plots at the center and the limb further illustrates the relative change in amplification at different wavelengths seen in Figure 3 - the amplification curve at the limb is virtually a vertical flip of the curve at the center. The overall slope of the curves indicates that for $\sigma_{0}=0$ the spectrum will appear bluer and for $\sigma_{0}=0.96$ redder than in the absence of a lens (see also Gould \& Welch 1996; Valls-Gabaud 1998).

The finer structure of the curves provides further interesting results. Sections of the curves with significant spectral variations of amplification indicate spectral regions particularly sensitive to microlensing. Observations of such regions during a transit event can then be used to test the depth dependence of the atmosphere model. While the resolution of this particular spectrum is too low to resolve individual spectral lines, broader molecular bands are clearly visible. An example of a sensitive feature is the $\mathrm{TiO}$ band system at $710 \mathrm{~nm}(A-X, \gamma$-bands), as hinted by the bump in the amplification curve. Recent models by Jacob et al. (1999) also point to the extreme limb-darkening effects in this band system. It should be noted that not all bands are affected in the same manner the $C-X \mathrm{TiO}$ system at $516 \mathrm{~nm}$ ( $\alpha$-bands) shows an opposite variation in amplification. The nature of the change in these bands is discussed in more detail further below.

The three amplification curves are each plotted for convenience with a different vertical scale. For example, the relative amplification variation at $\sigma_{0}=0.72$ is much smaller than at either the center or the limb. A simple way to quantify the degree of this variation is to take the maximum, minimum and average amplification over the full range of wavelengths and plot the ratio $\left(A_{\max }-A_{\min }\right) / A_{\text {aver }}$ as a function of lens position $\sigma_{0}$. This ratio in fact serves as a measure of chromaticity - it drops to zero in the achromatic point-source limit. Results for three different atmosphere models (wavelength range 401-775 nm) are presented in Figure 5. While there is a difference in overall amplitude, the general character of all three curves is similar. As the lens approaches the source, the degree of chromaticity increases, reaching a local peak of $\sim 3-5 \%$ just after crossing the limb. If the lens passes closer to the center, the chromaticity drops to $<0.5 \%$ at $\sigma_{0} \sim 0.7$ (approximately the M95-30 impact parameter, by coincidence). This indicates that the light curves for all the studied wavelengths intersect approximately at the same point, as seen in Figure 3 . A yet closer approach causes the chromaticity to increase to a peak value of $\sim 8-11 \%$ when the lens is positioned at the source center. To summarize, the strongest spectral 


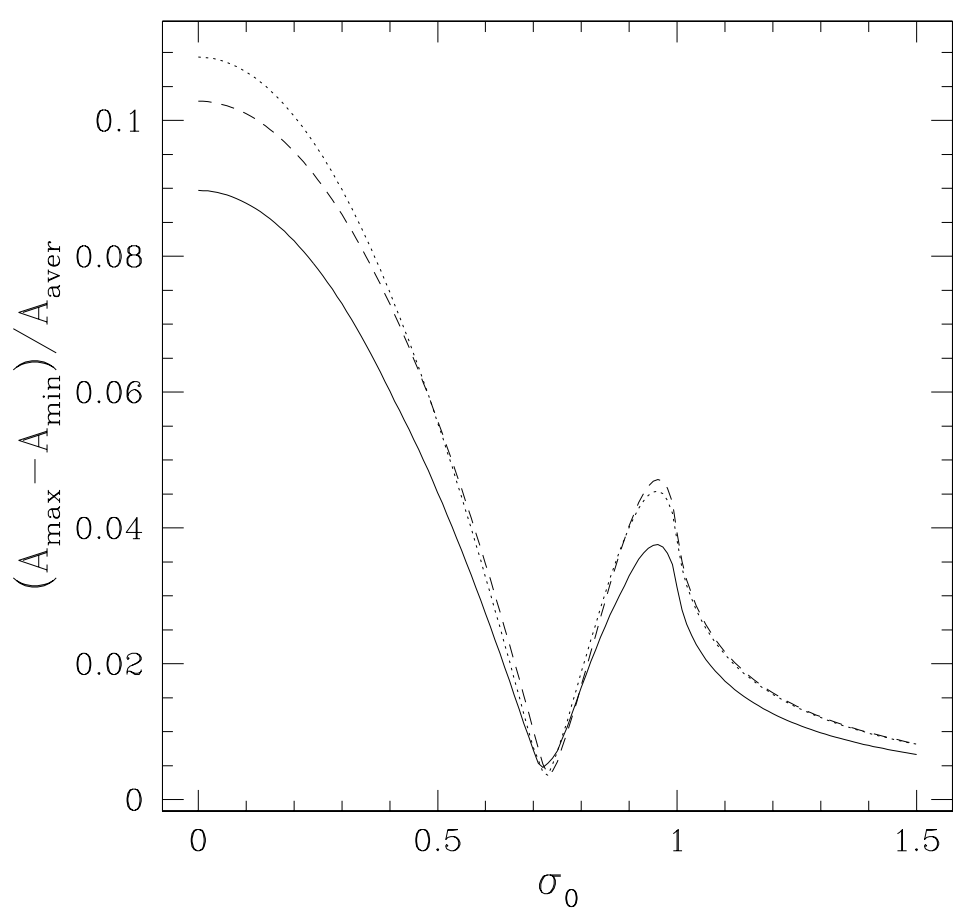

Fig. 5.- Variation of amplification within the low resolution spectrum as a function of lens position for three model atmospheres: $\mathrm{T}=3750 \mathrm{~K}, \log g=0.5$ (solid line), $\mathrm{T}=3500 \mathrm{~K}, \log g=1$ (dashed line) and $\mathrm{T}=4000 \mathrm{~K}, \log g=0.5$ (dotted line). The Einstein radius of the lens is the same as in previous figures $(\epsilon=13.23)$.

effects can be expected when the lens is aligned with the source center, with weaker effects near the limb. In between these two positions there is a drop in chromaticity nearly to zero (achromaticity). Although the limb effects are weaker, in principle they should be observable in any transit event.

The results in Figure 5 were computed using the M95-30 lens with $\epsilon=13.23$. The dependence of the results on the Einstein radius is explored in Figure 6 for the model atmosphere with $\mathrm{T}=3750 \mathrm{~K}$ and $\log g=0.5$. While the chromaticity predictably increases with $\epsilon$, its $\sigma_{0}$-dependence rapidly reaches a limit curve. In other words, the degree of lensing chromaticity cannot increase beyond this curve for a given atmosphere model and fixed spectral resolution. This behavior can be understood by studying the form of equation (5) during a close approach (as compared to the Einstein radius). In the limit $\epsilon \gg \sigma_{0}$ the

\footnotetext{
${ }^{2}$ Convergence requires also $\epsilon>\left(\sigma_{0}+1\right) / 2$.
} 


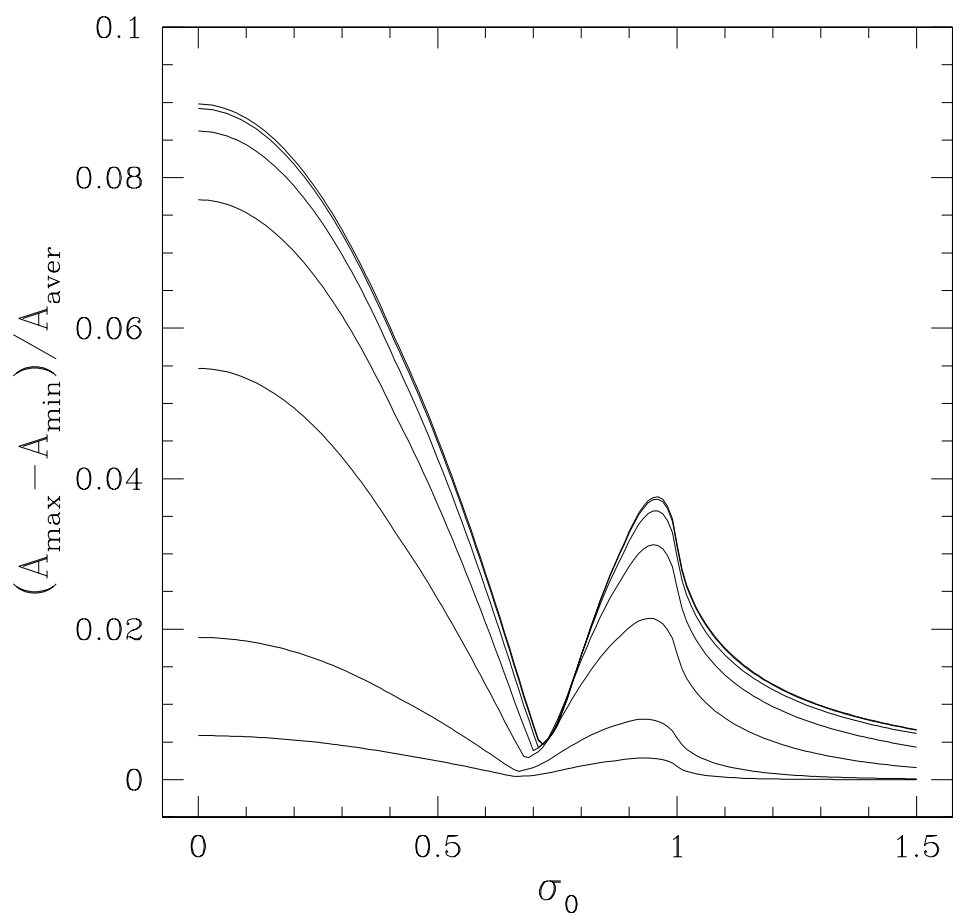

Fig. 6.- Variation of amplification within the low resolution spectrum (401-775 nm) as a function of lens position for the $\mathrm{T}=3750 \mathrm{~K}, \log g=0.5$ model atmosphere. Curves correspond to lenses with different Einstein radii in units of the source radius (from lowest curve): $\epsilon=$ $0.1,0.2,0.5,1,2,5,100$.

amplification

$$
A\left(\lambda, \sigma_{0}\right)=\epsilon \frac{\int_{\Sigma} \sigma^{-1} B(\lambda, \rho) d \Sigma}{\int_{\Sigma} B(\lambda, \rho) d \Sigma}+O\left(\frac{\sigma_{0}}{\epsilon}\right)
$$

is directly proportional to the Einstein radius $\epsilon$. The degree of chromaticity depends on amplification ratios and is therefore independent of $\epsilon$ in this limit, as seen in Figure 6. We conclude that as far as spectral effects are concerned, the "only" advantage of microlensing by stronger lenses $(\epsilon>5)$ is an overall increase in flux.

Figure 1 illustrates the capability of microlensing to resolve the depth structure of a given stellar atmosphere. Can the microlensing signature also be used to distinguish between different atmospheres? Figure 7 contains the results of Figure 4 together with those computed for the two other atmosphere models used in Figure 5; a cooler one ( $\mathrm{T}=3500 \mathrm{~K}$, $\log g=1)$ and a hotter one $(\mathrm{T}=4000 \mathrm{~K}, \log g=0.5)$. As expected, the results indeed vary substantially between the different models. In the $3500 \mathrm{~K}$ atmosphere, for example, the $\mathrm{TiO} \alpha$-bands at $516 \mathrm{~nm}$ show a stronger amplification variation, and more importantly, 

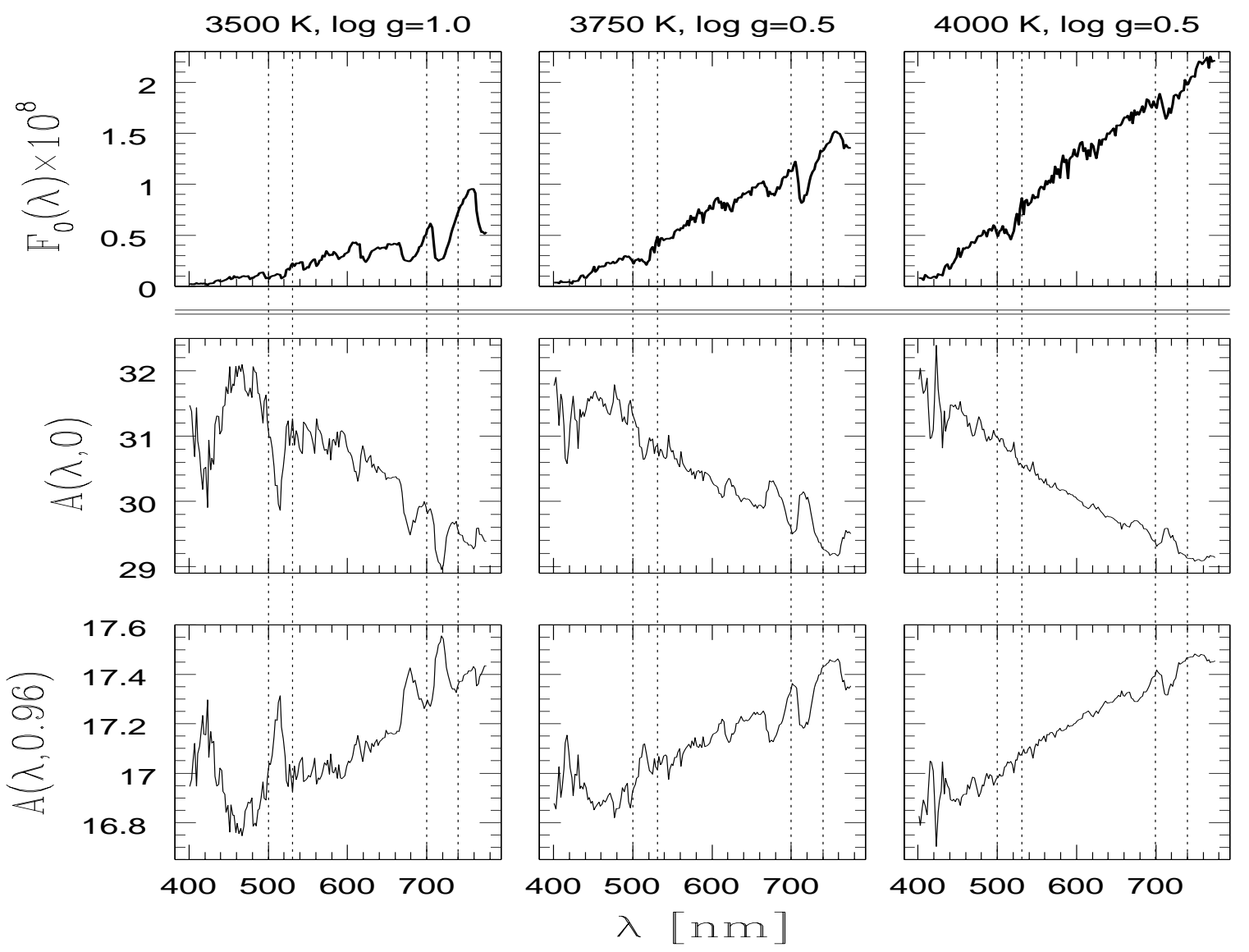

Fig. 7. - Microlensing by an $\epsilon=13.23$ lens of three different model atmospheres: $\mathrm{T}=3500 \mathrm{~K}$, $\log g=1.0$ (left column); $\mathrm{T}=3750 \mathrm{~K}, \log g=0.5$ (central column) and $\mathrm{T}=4000 \mathrm{~K}, \log g=0.5$ (right column). Upper row: low resolution unlensed spectra (flux in units of $\mathrm{W} \mathrm{m}^{-2} \mathrm{~Hz}^{-1}$ ); central row: amplification for lens position $\sigma_{0}=0$; lower row: amplification for lens position $\sigma_{0}=0.96$. Vertical dotted lines mark the $\mathrm{TiO} \alpha$-bands $(516 \mathrm{~nm})$ and $\gamma$-bands $(710 \mathrm{~nm})$.

the $\mathrm{TiO} \gamma$-bands at $710 \mathrm{~nm}$ show the opposite variation than in the $3750 \mathrm{~K}$ case. The amplification variation in the $4000 \mathrm{~K}$ model is much weaker overall, because the TiO bands are less prominent at this higher temperature. The only prominent amplification feature at $420 \mathrm{~nm}$ is in a spectral region with very low flux, hence it would be difficult to detect any spectral changes there.

\subsection{High Resolution Spectra and Line Profiles}



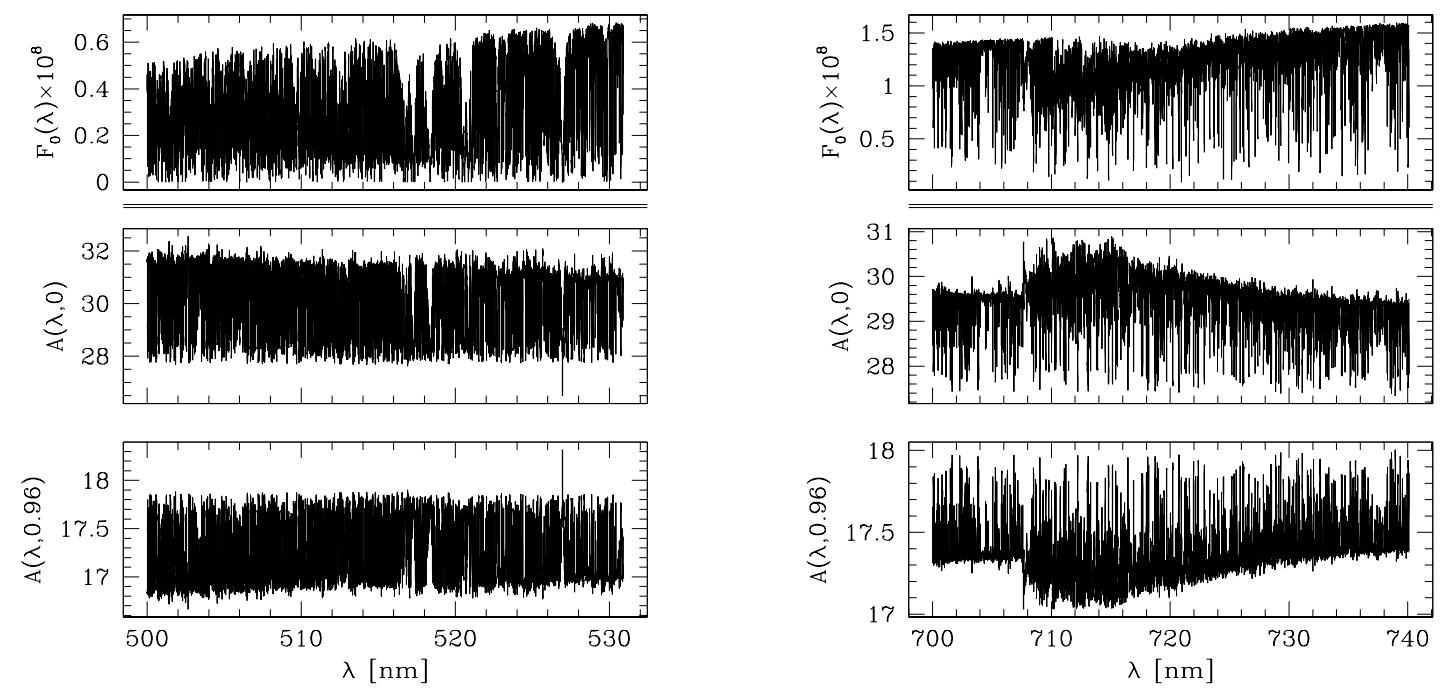

Fig. 8.- Upper row: high resolution unlensed spectrum of the $\mathrm{T}=3750 \mathrm{~K}, \log g=0.5$ model atmosphere (flux in units of $W \mathrm{~m}^{-2} \mathrm{~Hz}^{-1}$ ). Lower two rows: amplification by an $\epsilon=13.23$ lens as a function of wavelength for lens positions $\sigma_{0}=0$ and 0.96. Left panels: spectral region near the $516 \mathrm{~nm} \mathrm{TiO} \alpha$-bands. Right panels: spectral region near the $710 \mathrm{~nm} \mathrm{TiO}$ $\gamma$-bands.

The results presented in Figures 4, 5, 6 and 0 illustrate the overall microlensing effects on low resolution spectra. However, studying the behavior of finer features, such as individual molecular bands or spectral lines, can provide stronger constraints on model atmospheres. First we return to the $\mathrm{TiO}$ bands mentioned above. In this particular case, even the low resolution results illustrated in Figure 4 indicate the different behavior of the two band systems. When the lens is at $\sigma_{0}=0$, the $\gamma$-band system at $710 \mathrm{~nm}$ has a higher amplification in its region of highest absorption. As a result, the band system will appear weaker (shallower) than in the absence of the lens. With the lens at the limb, the highest absorption region has a lower amplification than the adjacent spectral region. The band system will therefore be more prominent than if the lens were absent. In contrast, the $\alpha$-band system at $516 \mathrm{~nm}$ shows exactly the opposite behavior. It will be more prominent with the lens at the source center, and less prominent when the lens is at the limb. The different behavior of the $\gamma$-band system is due to the extension of its region of formation higher in the atmosphere.

These effects are verified by the results of high spectral resolution $(\mathrm{R}=500,000)$ calculations shown in Figure 8. The first feature to notice in these plots, apart from the confirmed band system structure, is the high "fuzziness" of the amplification curves. This 


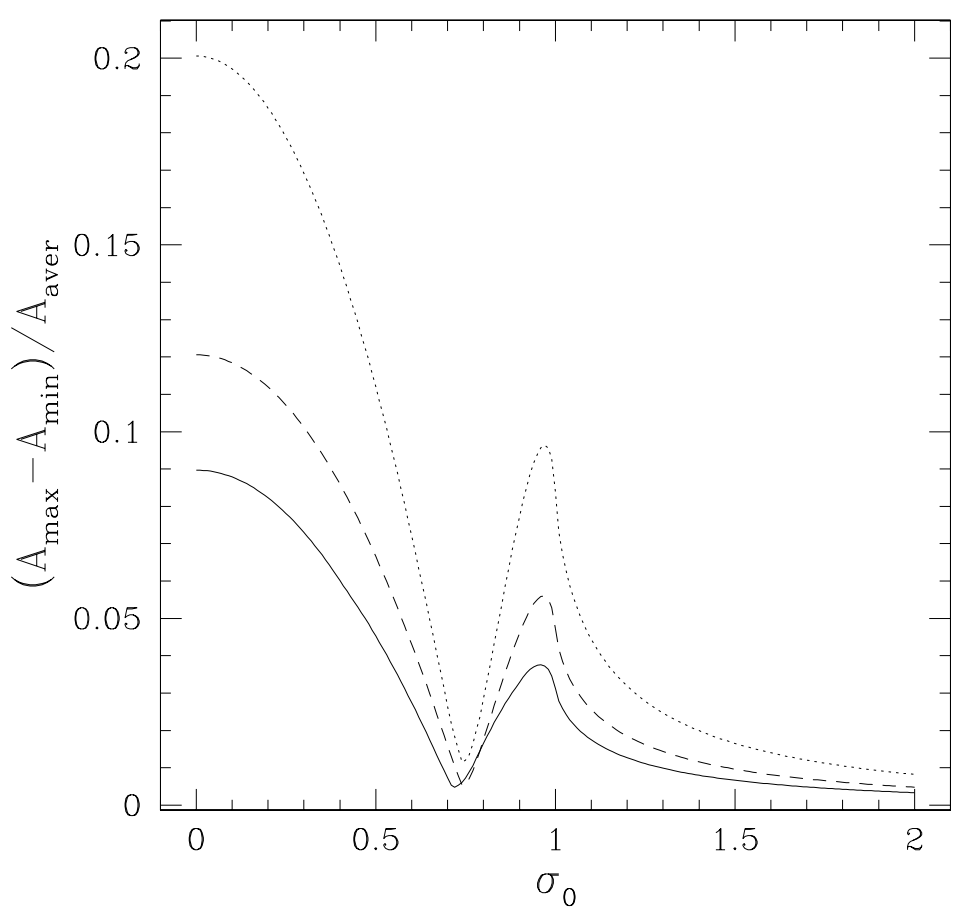

Fig. 9. - Variation of amplification as a function of lens position for the $\mathrm{T}=3750 \mathrm{~K}, \log g=0.5$ model atmosphere lensed by an $\epsilon=13.23$ lens. Low resolution spectrum 401-775 nm (solid line), high resolution 500-531 nm (dotted line) and high resolution 700-740 nm (dashed line).

property indicates that the amplification varies highly within individual spectral lines. In fact the less "fuzzy" $710 \mathrm{~nm}$ region also has a lower density of spectral lines. Studying the amplification variation of both regions in Figure 9, it comes as no surprise that while the shape of the dependence is similar, the degree of chromaticity is higher than in the full extent of the low resolution spectrum. This higher variation is due to the fine spectral structure, which is averaged out in the low resolution results. The degree of chromaticity for the $516 \mathrm{~nm}$ and $710 \mathrm{~nm}$ regions in high resolution again peaks at the source center $(20 \%$ and $12 \%$, respectively) and has a secondary peak at the limb (9.5\% and $5.5 \%$, respectively). For comparison, the center and limb values for the low resolution spectrum are $9 \%$ and $4 \%$, respectively. Note that the high values for the $516 \mathrm{~nm}$ region are partly caused by the single atomic spectral line at $526.95 \mathrm{~nm}$.

A closer look at the fine structure in both sets of high resolution results (apart from the large-scale $\mathrm{TiO}$ band effect) suggests a significant correlation between the unlensed

\footnotetext{
${ }^{3}$ Unfortunately this line is highly saturated at its core, where the amplification undergoes the high variation - the effect will therefore be difficult to detect because of the low photon flux at the line center.
} 

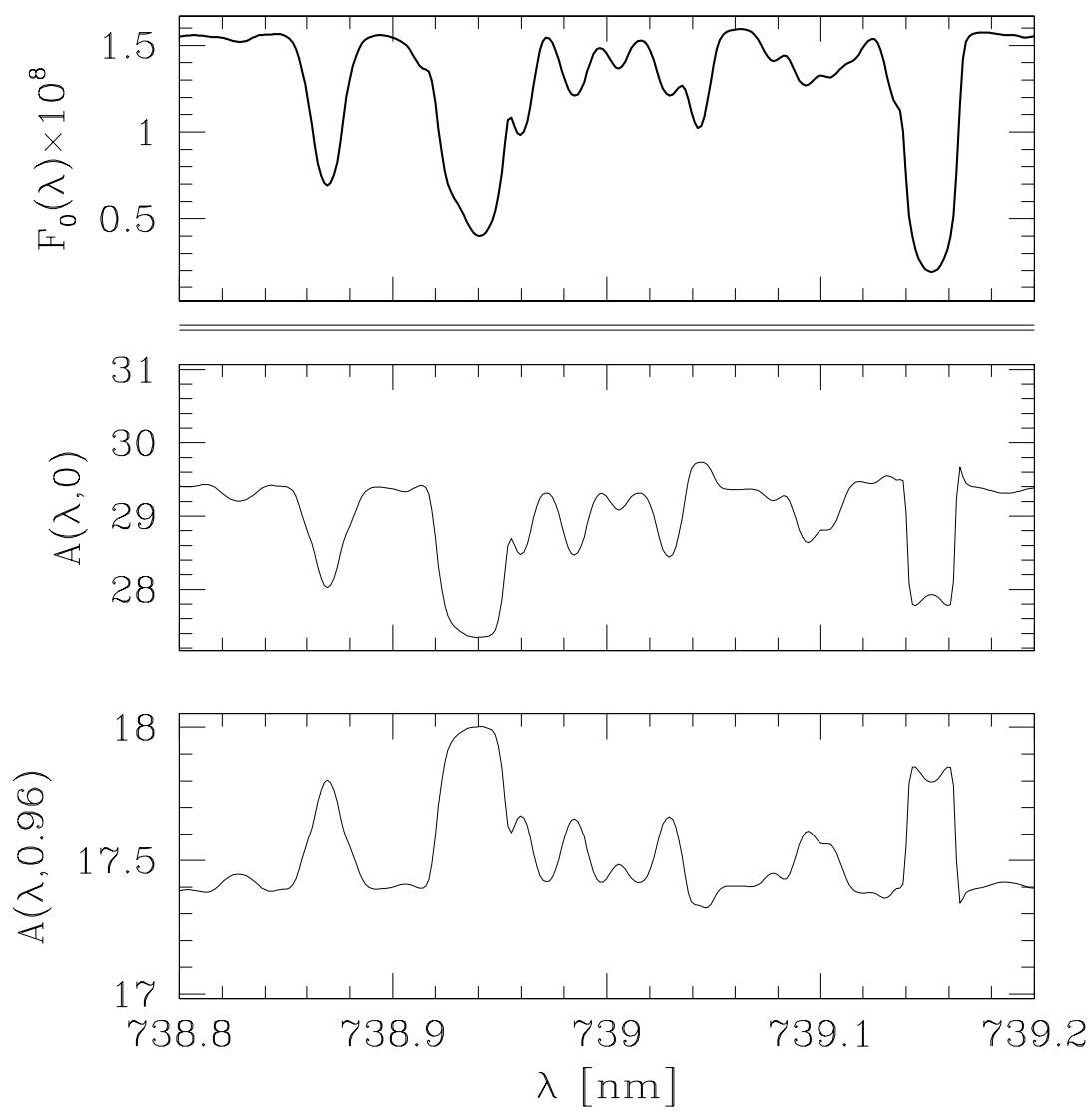

Fig. 10.- Detail of Figure 8 resolving individual spectral lines. The spectral region shown is between $738.8-739.2 \mathrm{~nm}$.

spectrum and the amplification curve for $\sigma_{0}=0$. A blow-up of a $0.4 \mathrm{~nm}$ interval in Figure 10 confirms the correlation - most individual lines have their cores amplified less than their wings at $\sigma_{0}=0$, while at $\sigma_{0}=0.96$ the cores are amplified more than the wings. Hence most lines become more prominent with the lens at the source center, and less prominent with the lens close to the limb than in the absence of the lens $\left(\sigma_{0} \rightarrow \infty\right)$. There are fewer examples of lines with the opposite behavior (e.g. at $739.044 \mathrm{~nm}$ in Figure 10), with virtually no change (flat amplification curve) or with more complex behavior.

The characteristic correlation can be explained for typical absorption lines in the following way. Such lines form at a certain depth in the atmosphere, and gradually become less prominent away from the center towards the limb, as there is relatively less absorption at the limb. The center-to-limb variation curves at the cores of these lines are therefore flatter than in the adjacent continuum. The correlation is now directly explained by the discussion of light curve shapes above - when the lens is at the source center, the line cores are amplified less than the continuum, while with the lens at the limb the cores are 

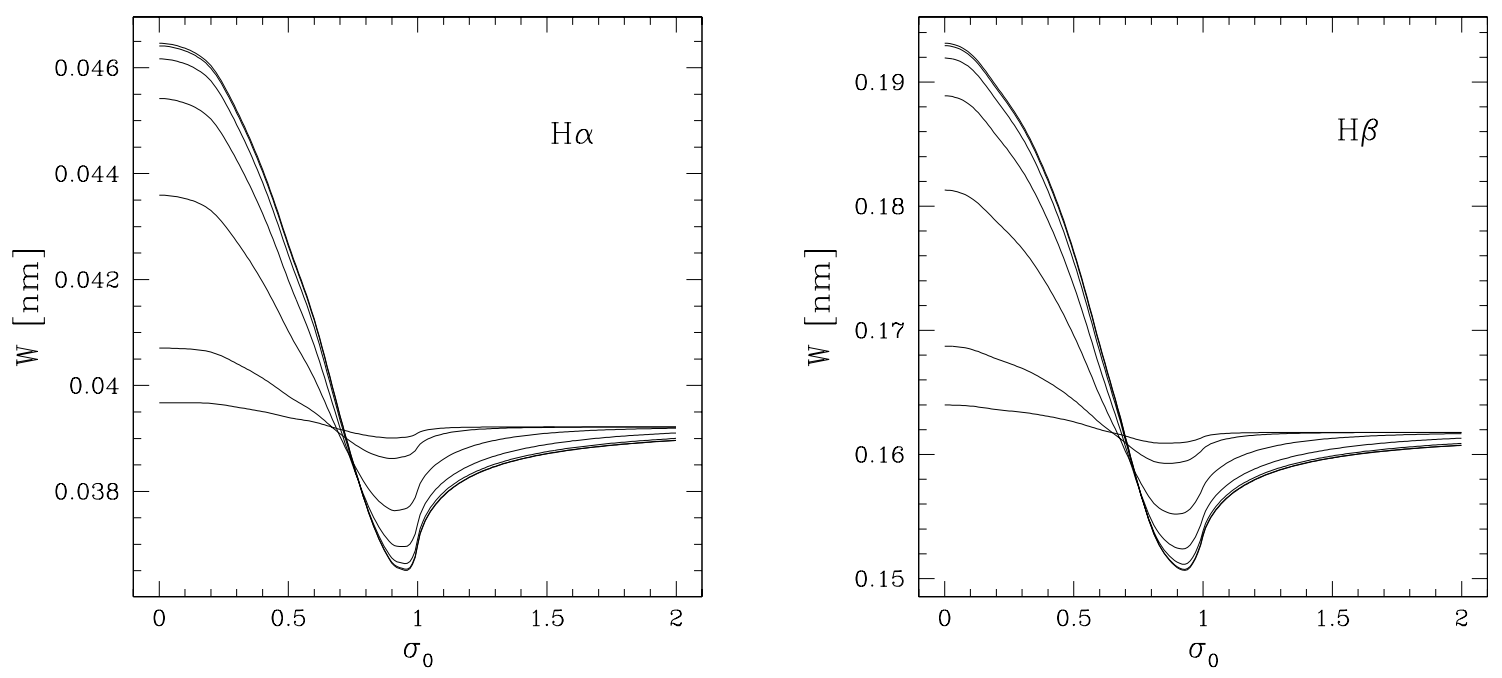

Fig. 11. - Equivalent width of $\mathrm{H} \alpha$ (left panel) and $\mathrm{H} \beta$ (right panel) as a function of lens position, for the $\mathrm{T}=3750 \mathrm{~K}, \log g=0.5$ model atmosphere. Individual curves correspond to different lenses (from lowest at $\sigma_{0}=0$ ): $\epsilon=0.1,0.2,0.5,1,2,5,100$.

amplified more than the continuum.

The less common anti-correlation (or lack of correlation) of the unlensed spectrum and the amplification at source center can be expected for example in some emission features, high excitation lines, or in spectral lines affected by more complex physical effects, such as resonant scattering (Loeb \& Sasselov 1995), overlapping lines, lines within molecular bands, etc.

A straightforward method for studying the change of a particular line is to compute its equivalent width, which is generally given by

$$
W\left(\sigma_{0}\right)=\Delta\left[1-\frac{\int F\left(\lambda, \sigma_{0}\right) d \lambda}{\int F_{c}\left(\lambda, \sigma_{0}\right) d \lambda}\right],
$$

where $F_{c}\left(\lambda, \sigma_{0}\right)$ is the continuum flux and the integrals are performed over a wavelength interval of width $\Delta$ around the line. As an example we present here results for the hydrogen Balmer lines $\mathrm{H} \alpha$ and $\mathrm{H} \beta$ in the same $\mathrm{T}=3750 \mathrm{~K}, \log g=0.5$ model. The center-to-limb variation of their unlensed profiles is shown in Figure 11. The dependence of their equivalent widths on lens position is plotted in Figure 11 for a set of Einstein radii $\epsilon$. First we note that the change with $\epsilon$ has the same character as in Figure 6. Any sufficiently strong lens $(\epsilon>5)$ will therefore affect the equivalent width in the same way. This result can again be traced to the fact that the equivalent width in equation (8) depends on a ratio of 
amplifications. This ratio is independent of $\epsilon$ in the $\epsilon \gg \sigma_{0}$ limit, as seen from equation (7). During a microlensing event both Balmer lines behave in the typical manner described above. An approach of a sufficiently strong lens first causes the width of both lines to drop (by $\sim 7 \%$ ), as it gives higher weight to the limb where the lines are weak. Similarly, a lens positioned at the center, where both lines are strong, would increase their width $(\sim 19 \%)$ above the asymptotic value. Both the $\mathrm{H} \alpha$ and $\mathrm{H} \beta$ curves are similar, although those of $\mathrm{H} \beta$ are more centrally peaked and have a broader minimum near the limb.

We compared our results to those of Valls-Gabaud (1998) for $\mathrm{H} \beta$ with the same event parameters $\mathrm{f}$. The general character of the change with lens position is the same - decrease of the equivalent width (EW) at the limb and a higher increase closer to the source center. However, Valls-Gabaud's results show a two to three times lower relative change of the EW, the actual factor depending on the lens position. As a result, the predicted ratio of EW increase (close to the center) to peak EW decrease (at the limb) is also lower than our computations show. Furthermore, the achromaticity point appears to be closer to the limb (around $\sigma_{0}=0.8$ ) than in our analysis. As mentioned in $\S 2$, assumptions such as linear limb darkening degrade Valls-Gabaud's models too much to give realistic predictions of equivalent widths, particularly in the case of red giants. This comparison further illustrates the sensitivity of microlensing to the atmosphere structure of the resolved source.

A detailed study of high resolution spectral changes in different model atmospheres and comparison with the M95-30 observational results will be presented elsewhere (Heyrovský \& Sasselov 2000b).

\section{Conclusions}

Microlensing events in which a point-mass lens with Einstein radius $\epsilon>4$ (in source radius units) approaches the source star within three source radii already exhibit $\sim 1 \%$ deviations from point-source light curves. The deviations further increase for closer approaches. These finite source effects depend on the surface brightness distribution of the source star. Observations of such effects, particularly during source transit events, can therefore be used to resolve and study the structure of the stellar disk. As the brightness distribution depends on wavelength, the usual assumption of microlensing

4 We neglect here the earlier results in Valls-Gabaud (1996), which are inconsistent with VallsGabaud (1998). 
achromaticity breaks down in these cases. Spectral observations can then be used to probe the depth structure of the atmosphere of the source star. The most promising sources for such studies are red giants in the Galactic bulge, among other reasons due to their large size, intrinsic brightness and fairly high abundance. These circumstances are in fact particularly fortuitous, as the structure of cool red giant atmospheres is currently very poorly observationally constrained.

The degree of microlensing chromaticity can be measured by the relative spectral variation of the microlensing amplification. Thus defined, chromaticity as a function of lens position is found to have two peaks - primary peak at the source center and secondary peak close to the source limb, with a dip to achromaticity in between. In the usually observed type of events, in which the Einstein radius $\epsilon>5$, chromaticity is independent of $\epsilon$. Its peak values for a low resolution optical spectrum are typically $10 \%$ at the center and $4 \%$ at the limb (both values increase with higher spectral resolution). Any such transit event should therefore exhibit at least the $4 \%$ effect, which should be readily observable under favorable conditions. In future potential source-crossing events it is therefore important not to miss the time when the lens is close to the limb, as was unfortunately the case in M95-30.

During a red giant transit event the overall spectrum appears redder when the lens is at the source limb, and bluer if the lens comes close to the source center. Individual spectral lines respond to microlensing in different ways, because of their different center-to-limb variation, which reflects their different depth of formation in the star's atmosphere. Most absorption lines will typically turn weaker (lower equivalent width) when the lens is close to the limb and more prominent when the lens is near the center. Some emission lines, high excitation lines, overlapping lines, lines within molecular bands and lines affected by more complex physical effects can exhibit opposite or generally different behavior. As a result, broad molecular bands visible even on a low resolution spectrum of a given source may behave in different ways. The pattern of their changes depends on physical parameters, composition and structure of the atmosphere. Observations of these bands can therefore be used to constrain the global parameters, and to check theoretical assumptions used for constructing model stellar atmospheres. High resolution spectra resolving individual lines would, however, also enable studies of the depth structure of the star's atmosphere in detail. Particularly in the case of red giants, gravitational microlensing therefore provides a unique new tool for stellar physics.

During M95-30, the only well-documented point-mass microlensing transit event up to now, eleven low resolution and three high resolution spectra were taken in addition to photometry in two colors (Alcock et al. 1997d). In a forthcoming paper (Heyrovský \& Sasselov 2000b), we will use the methods and findings described here together with 
the observational data to study the lensed giant and construct an adequate model of its atmosphere.

We thank R. Kurucz for his kind help with opacities and molecular data. We also thank an anonymous referee for comments that helped improve the manuscript. DS is grateful to I. Shapiro for his support during part of this study.

\section{REFERENCES}

Afonso, C., et al. 1999, A\&A, 344, L63

Albrow, M., et al. 1999, ApJ, 522, 1011

Alcock, C., et al. 1997a, ApJ, 479, 119

Alcock, C., et al. 1997b, ApJ, 486, 697

Alcock, C., et al. 1997c, ApJ, 491, L11

Alcock, C., et al. 1997d, ApJ, 491, 436

Andersen, J. 1991, A\&A Review, 3, 91

Armstrong, J. T., et al. 1995, Physics Today, 48, 42

Becker, A., et al. 1998, BAAS, 30, 1415

Bessell, M. S., Brett, J. M., Scholz, M., \& Wood, P. R. 1989, A\&AS, 77, 1

Bessell, M. S., Brett, J. M., Scholz, M., \& Wood, P. R. 1991, A\&AS, 89, 335

Bogdanov, M. B., \& Cherepashchuk, A. M. 1995, ARep, 39, 779

Bogdanov, M. B., \& Cherepashchuk, A. M. 1996, ARep, 40, 713

Bogdanov, M. B., Cherepashchuk, A. M., \& Sazhin, M. V. 1996, Ap\&SS, 235, 219

Gaudi, B. S., \& Gould, A. 1999, ApJ, 513, 619

Gould, A. 1994, ApJ, 421, L71

Gould, A. 1995, ApJ, 446, L71

Gould, A. 1997, ApJ, 483, 98

Gould, A., \& Welch, D. L. 1996, ApJ, 464, 212

Hauschildt, P. H., Allard, F., Alexander, D. R., \& Baron, E. 1997, ApJ, 488, 428

Hauschildt, P. H., Allard, F., Ferguson, J., Baron, E., \& Alexander, D. R. 1999, ApJ, 525, 871 
Hendry, M. A., Coleman, I. J., Gray, N., Newsam, A. M., \& Simmons, J. F. L. 1998, NewAR, 42, 125

Heyrovský, D., \& Loeb, A. 1997, ApJ, 490, 38 (Paper I)

Heyrovský, D., \& Sasselov, D. D. 2000a, ApJ, in press

Heyrovský, D., \& Sasselov, D. D. 2000b, in preparation

Hofmann, K.-H., \& Scholz, M. 1998, A\&A, 335, 637

Houdashelt, M. L., Bell, R. A., Sweigart, A. V., \& Wing, R. F. 2000, AJ, in press, preprint (astro-ph/9911383)

Ignace, R., \& Hendry, M. A. 1999, A\&A, 341, 201

Jacob, A. P., Bedding, T. R., Robertson, J. G., \& Scholz, M. 1999, MNRAS, in press, preprint (astro-ph/9911076)

Kurucz, R. L. 1992, in IAU Symp. 149, The Stellar Populations of Galaxies, ed. B. Barbuy \& A. Renzini (Dordrecht: Kluwer), 225

Kurucz, R. L. 1999, in IAU Gen.Ass. XXIII, Highlights of Astronomy Vol.11B, ed. J. Andersen (Dordrecht: Kluwer), 646

Lennon, D. J., Mao, S., Fuhrmann, K., \& Gehren, T. 1996, ApJ, 471, L23

Loeb, A., \& Sasselov, D. 1995, ApJ, 449, L33

Magain, P. 1986, A\&A, 163, 135

Maoz, D., \& Gould, A. 1994, ApJ, 425, L67

Mozurkewich, D., et al. 1991, AJ, 101, 2207

Nemiroff, R. J., \& Wickramasinghe, W.A.D.T. 1994, ApJ, 424, L21

Paczyński, B. 1996, ARA\&A, 34, 419

Plez, B., Brett, J. M., \& Nordlund, A. 1992, A\&A, 256, 551

Renault, C., et al. 1997, A\&A, 324, L69

Sasselov, D. D. 1996, in ASP Conf. Ser. 109, Cool Stars 9, ed. R. Pallavicini \& A. K. Dupree (San Francisco: ASP), 541

Scholz, M., \& Tsuji, T. 1984, A\&A, 130, 11

Schwenke, D. W. 1998, in Faraday Discussions 109, Chemistry and Physics of Molecules and Grains in Space, ed. The Faraday Division of the Royal Society of Chemistry (London: Royal Society of Chemistry), 321

Simmons, J. F. L., Newsam, A. M., \& Willis, J. P. 1995, MNRAS, 276, 182 
Simmons, J. F. L., Willis, J. P., \& Newsam, A. M. 1995, A\&A, 293, L46

Udalski, A., et al. 1997, Acta Astron., 47, 169

Uitenbroek, H., Dupree, A. K., \& Gilliland, R. L. 1998, AJ, 116, 2501

Valls-Gabaud, D. 1995, in Large Scale Structure in the Universe, ed. J. P. Mücket, S. Gottlöber, \& V. Müller (Singapore: World Scientific), 326

Valls-Gabaud, D. 1996, in IAU Symp. 173, Astrophysical Applications of Gravitational Lensing, ed. C. S. Kochanek \& J. N. Hewitt (Dordrecht: Kluwer), 237

Valls-Gabaud, D. 1998, MNRAS, 294, 747

Vandenberg, D., \& Bell, R. A. 1985, ApJS, 58, 561

Witt, H. J. 1995, ApJ, 449, 42

Witt, H. J., \& Mao, S. 1994, ApJ, 430, 505 